\title{
High mobility group protein A2 overexpression indicates poor prognosis for cancer patients: a meta-analysis
}

\author{
Dan Nie ${ }^{1}$, Lingping Zhang ${ }^{2}$, Qian Guo ${ }^{1}$ and Xiguang $\mathrm{Mao}^{1}$ \\ ${ }^{1}$ Department of Gynecology, The Affiliated Hospital of Southwest Medical University, Luzhou, Sichuan, People's Republic of \\ China \\ ${ }^{2}$ Department of Neonatology, The Affiliated Hospital of Southwest Medical University, Luzhou, Sichuan, People's Republic of \\ China
}

Correspondence to: Dan Nie, email: niedan@swmu.edu.cn

Keywords: high-mobility group protein A; prognosis; meta-analysis

Received: July 29, $2017 \quad$ Accepted: November 17, $2017 \quad$ Published: December 10, 2017

Copyright: Nie et al. This is an open-access article distributed under the terms of the Creative Commons Attribution License 3.0 (CC BY 3.0), which permits unrestricted use, distribution, and reproduction in any medium, provided the original author and source are credited.

\section{ABSTRACT}

Overexpression of the high mobility group protein A2 (HMGA2), an architectural transcription factor, has been linked to poor prognosis in many malignancies, although this remains controversial. Herein, we conducted a meta-analysis to investigate whether HMGA2 has prognostic value, and evaluated the association between HMGA2 and clinicopathologic factors in malignancies. A total of 29 studies involving 4114 patients were included in this meta-analysis. The pooled results demonstrated that elevated HMGA2 predicted a poor overall survival (OS) (hazard ratio $[H R]=1.82$; $95 \%$ confidence interval $[\mathrm{CI}]=1.62-2.05 ; P<0.001)$ and disease-free survival/ progression-free survival/recurrence-free survival $(H R=1.94 ; 95 \% \mathrm{CI}=1.27-2.98$; $P=0.002$ ). Subgroup analysis conducted by study region, sample size, detection method, and analysis method indicated that HMGA2 overexpression correlated with poor OS. Furthermore, HMGA2 overexpression was found to be linked to poor OS in various cancers except ovarian cancer (pooled HR $=1.14 ; 95 \% \mathrm{CI}=0.62-2.09 ; P=$ 0.673). High HMGA2 expression level also correlated with advanced TNM stage (OR $=2.44 ; 95 \% C I=1.87-3.2 ; P<0.001)$, lymphovascular invasion $(O R=2.46,95 \%$ $C I=1.67-3.64 ; P<0.001)$, distant metastasis (OR = 2.66; 95\% CI $=1.51-4.69 ; P<$ 0.001 ), and lymph node metastasis ( $O R=1.83 ; 95 \% C I=1.27-2.64 ; P=0.001)$. In conclusion, HMGA2 overexpression indicates a worse prognosis and may serve as a prognostic predictor in cancer patients.

\section{INTRODUCTION}

Cancer is a major public health problem worldwide and the leading cause of death in China [1]. Based on the GLOBOCAN estimates of cancer morbidity and mortality, approximately 14.1 million new cases and 8.2 million deaths occurred in 2012 [2]. The number of cancer survivors has increased steadily because of the advances in early detection and treatment as well as the aging and growth of the population [3]. However, the 5-year survival rate of most cancers is still low, many patients are asymptomatic and often diagnosed in an advanced disease stage [4]. Therefore, it is necessary to identify new prognostic and diagnostic markers that can help clinicians identify patients with a poor prognosis and use more efficient treatment strategies.

High-mobility group AT-hook 2 (HMGA2), a member of the high mobility group (HMG) protein family, is a non-histone chromosomal protein [5, 6]. It modulates gene transcription by interacting with various transcription factors and altering the chromatin structure [6,7], and is a known regulator of cell growth, differentiation, apoptosis, and DNA repair $[8,9]$. High expression of HMGA2 has been detected in both epithelial and mesenchymal tissue 
of malignant tumors, and may promote tumorigenesis [9]. Recent studies have revealed that the overexpression of HMGA2 correlates with higher lymph node metastasis rates, poor tumor differentiation, and unfavorable prognosis [10-14], implying that HMGA2 has prognostic value in cancer.

Recently, a meta-analysis in gastric cancer indicated that HMGA2 overexpression is associated with poor prognosis of cancer patients [15]. However, only six studies involving 712 gastric cancer patients were analyzed in this study. Moreover, whether overexpression of HMGA2 has prognostic value in other cancer types has not been evaluated. Therefore, we conducted a metaanalysis of relevant studies to understand if HMGA2 has predictive prognostic potential in various cancers.

\section{RESULTS}

\section{Characteristics of eligible studies}

A total of 696 articles were identified after the primary search, of which 304 duplicate articles were removed, and a further 301 articles were excluded after reviewing the title and abstract. The full texts of the remaining 91 articles were reviewed, and additional exclusions were made: 32 studies: survival data not reported; 6 studies: insufficient data for quantitative analysis, 10 studies: not written in English; 14 studies: conference abstracts or reviews; and 2 studies: involved animal experiments. Finally, 27 qualifying studies were enrolled for this meta-analysis (Figure 1).

Among the 27 articles, two studies included two different cohorts with survival data, so that 29 studies including a total number of 4114 patients were analyzed. These studies were mainly conducted in East Asia, Europe, and the United States of America. The cancer types studied were: gastric cancer (GC) [16-18], breast cancer (BC) $[10,19]$, hepatocellular carcinoma (HCC) $[11,13]$, colorectal cancer (CRC) $[14,20,21]$, ovarian cancer (OC) $[22,23]$, nasopharyngeal carcinoma (NPC) $[24,25]$, esophageal carcinoma (EC) [26, 27], head and neck squamous cell carcinoma (HNSCC) [28-30], clear cell renal cell carcinoma [12], intrahepatic cholangiocarcinoma [31], glioblastoma [32], gallbladder adenocarcinoma [33], melanoma [34], non-small-cell lung cancer [35], and bladder cancer [36]. HMGA2 was detected using immunohistochemistry (IHC) (24 studies), reverse transcription-polymerase chain reaction (RT-PCR) (2 studies), or quantitative realtime polymerase chain reaction (qRT-PCR) ( 3 studies). The expression of HMGA2 was correlated with overall survival (OS) in 25 studies, and disease-free survival (DFS)/progression-free survival (PFS)/recurrence-free survival (RFS) in 4 studies. Studies were assessed using the Newcastle-Ottawa Scale (NOS). Supplementary Table 1 displays the general characteristics of the qualifying studies.

\section{Correlation between HMGA2 expression and OS}

Combined analysis of 25 studies suggested that the overexpression of HMGA2 correlated with poor OS of cancer patients (pooled hazard ratio $[\mathrm{HR}]=1.82 ; 95 \%$ confidence interval $[\mathrm{CI}]=1.62-2.05 ; P<0.001)$. Low heterogeneity was observed between these studies $\left(I^{2}=\right.$ $16.4 \% ; P=0.231$; fixed effects) (Figure 2). Subgroup analysis was conducted based on study region, sample size, detection method, analysis method, and cancer type (Table 1). Subgroup analysis by cancer type showed that high HMGA2 expression was associated with worse OS in GC (pooled HR $=1.77 ; 95 \% \mathrm{CI}=1.31-2.41 ; P<0.001$ ), $\mathrm{BC}$ (pooled $\mathrm{HR}=2.26 ; 95 \% \mathrm{CI}=1.56-3.28 ; P<0.001)$, HCC (pooled HR $=1.90 ; 95 \% \mathrm{CI}=1.37-2.64 ; P<0.001$ ), CRC (pooled HR $=1.78 ; 95 \% \mathrm{CI}=1.29-2.44 ; P<0.001$ ), NPC (pooled HR $=1.96 ; 95 \% \mathrm{CI}=1.26-3.05 ; P=0.003)$, and $\mathrm{EC}$ (pooled $\mathrm{HR}=1.82 ; 95 \% \mathrm{CI}=1.19-2.77 ; P=0.006$ ). However, in ovarian cancer (pooled $\mathrm{HR}=1.14 ; 95 \% \mathrm{CI}=$ 0.62-2.09; $P=0.673)$, HMGA2 overexpression did not prognosticate OS. In subgroup analysis by study region, patients from both Asian $(\mathrm{HR}=1.95 ; 95 \% \mathrm{CI}=1.69-2.25$; $P<0.001)$ and non-Asian regions $(\mathrm{HR}=1.60 ; 95 \% \mathrm{CI}=$ $1.14-2.25 ; P=0.007)$ showed a significant correlation between high HMGA2 expression and poor OS. Pooled HR results for subgroup analyses by sample size, detection method and analysis method were $>1$ in all subgroups.

\section{Correlation between HMGA2 expression and clinicopathological features}

The correlation between HMGA2 expression and clinicopathologic features is shown in Figure 3A-3D. High HMGA2 expression was related to advanced tumor node metastasis (TNM) stage (stage III/IV) (odds ratio $[\mathrm{OR}]=2.44 ; 95 \% \mathrm{CI}=1.87-3.2 ; P<0.001$ ), positive lymphovascular space invasion $(\mathrm{OR}=2.46,95 \% \mathrm{CI}$ $=1.67-3.64 ; P<0.001)$, distant metastasis $(\mathrm{OR}=2.66$; $95 \%$ CI $=1.51-4.69 ; P<0.001)$, and lymph node metastasis $(\mathrm{OR}=1.83 ; 95 \% \mathrm{CI}=1.27-2.64 ; P=0.001)$.

\section{Correlation between HMGA2 expression and DFS/PFS/RFS}

Analysis of data pooled from seven studies that reported DFS/PFS/RFS (three of which included both OS and DFS/PFS/RFS data) showed that overexpression of HMGA2 predicted a poor DFS/PFS/RFS in the random effects model $(\mathrm{HR}=1.94 ; 95 \% \mathrm{CI}=1.27-2.98 ; P=$ $0.002)$. A high degree of heterogeneity was detected among the studies $\left(I^{2}=58.8 \%, P=0.024\right)$ (Figure 4). Subgroup analysis was not performed due to the small number of studies in this category.

\section{Publication bias and sensitivity analysis}

Begg's test and Egger's test were applied to assess publication bias. The results indicated that there 
was no obvious publication bias for OS $(P=0.199$ for Begg's test and 0.271 for Egger's test) or DFS/PFS/RFS ( $P=0.764$ for Begg's test and $P=0.076$ for Egger's test) among the included studies (Figure $5 \mathrm{~A}$ and $5 \mathrm{~B}$ ). To ensure stability of results, we performed sensitivity analysis by sequentially omitting each study and analyzing the remaining datasets. We found that none of the studies had a significant effect on the OS or DFS/PFS/RFS (Figure 6A and $6 \mathrm{~B}$ ) independent of the others. This confirmed that the outcomes were stable and reliable.

\section{DISCUSSION}

The HMGA protein family contains HMGA1a, HMGA1b, HMGA1c, and HMGA2 proteins [37]. Recently, evidence has implicated HMGA2 having complex functions in cancer [9]. HMGA2 promotes tumorigenesis through regulating transcription of human telomerase reverse transcriptase (hTERT) and binding directly to the promoters of FN1 and IL11 $[38,39]$. Moreover, aberrant expression of HMGA2 promotes cancer invasion, metastasis, and epithelialto-mesenchymal transition (EMT) by activating the transforming growth factor beta $(\mathrm{TGF} \beta)$ and Wnt/ $\beta$ catenin signaling pathways $[40,41]$. In addition, HMGA2 through activating HMGA2-FOXL2-ITGA2 and HMGA2-TET1-HOXA9 pathways prompts cancer distant metastasis and chemoresistance [42, 43]. Furthermore, miRNAs like miR-490-3p and miR-145 can inhibit cancer development and progression by direct regulation of HMGA2 expression [23, 44]. A study by Li et al. [45] showed that long non-coding ribonucleic acid (lncRNA) HIT000218960 promotes papillary thyroid cancer by upregulating HMGA2 expression. These results suggest that HMGA2 acts as a target gene of many micro RNAs (miRNAs) and lncRNAs, which play critical roles in regulating tumorigenesis and cancer progression. In addition, several recent studies demonstrated that HMGA2 overexpression, both in the tissues and blood of patients with cancer correlated with poor tumor differentiation, positive lymph node metastasis, and advanced stage, indicating a poor prognosis $[46,47]$. As a member of the

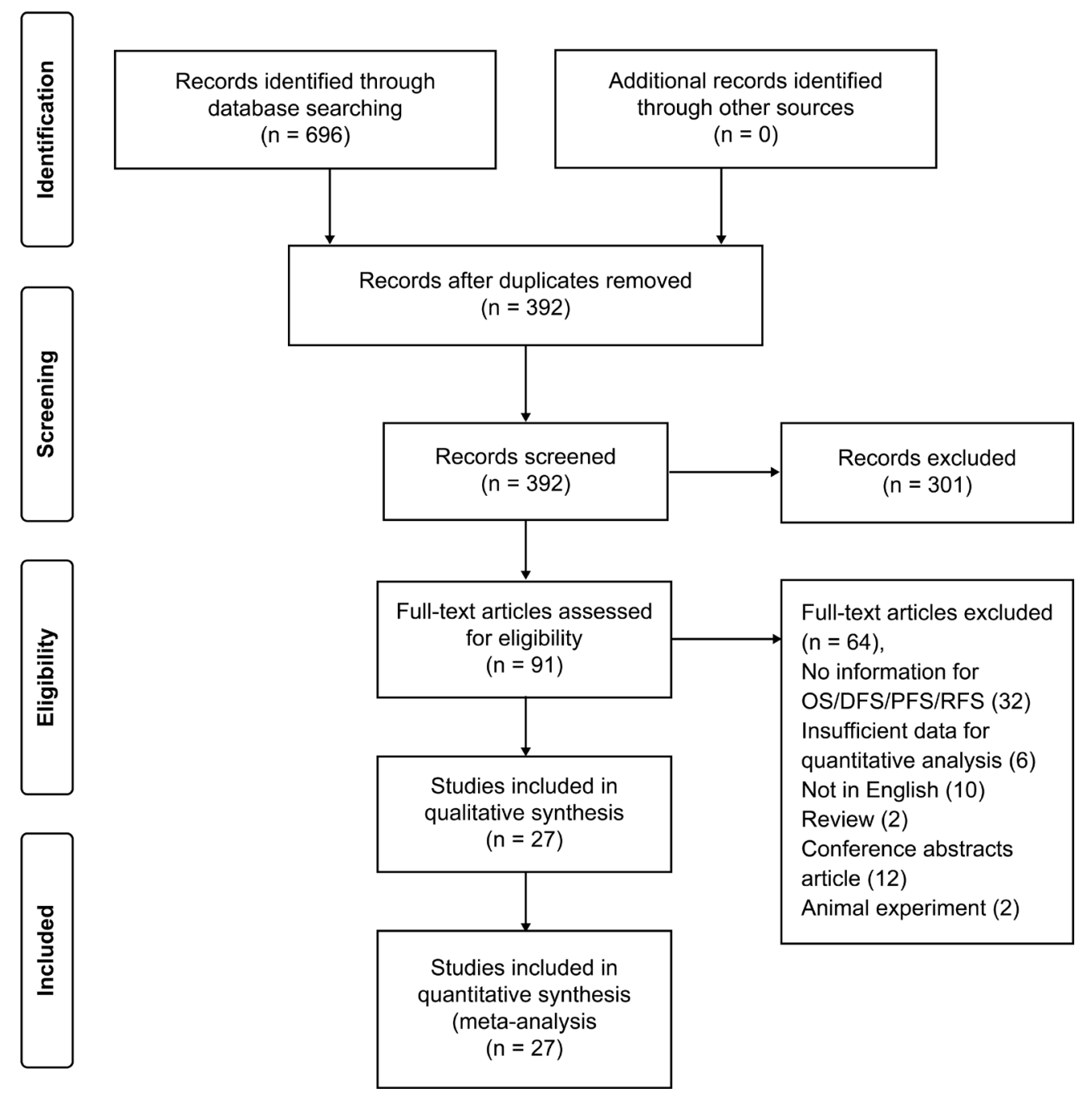

Figure 1: Flow chart of study selection strategy. 
HMGA protein family, HMGA1 also has been reported to be overexpressed in cancers and promote tumorigenesis [48-51]. However, the prognostic role of HMGA1 in different types of cancers remains controversial. Liau et al. [52] suggested that HMGA1 overexpression promotes tumorigenicity through activating PI3-K/Akt-dependent signaling pathways and predicts poor postoperative survival of pancreatic adenocarcinoma patients. While Jun et al. [18] indicated that HMGA1 overexpression was not correlated with lymphatic invasion, TNM stage, or cancer recurrence. Therefore, further studies are needed to clarify the prognostic role of HMGA1 in different types of cancers. In this meta-analysis, we only sought to understand whether HMGA2 can be used as a prognostic biomarker in cancer patients.

In this meta-analysis, we included 4114 patients from 29 studies, and the outcomes demonstrated a statistically significant correlation between high HMGA2 expression and poor OS. Meanwhile, high HMGA2 expression was significantly correlated with short DFS/PFS/RFS. In subgroup analysis, we found that high expression of HMGA2 conferred a worse OS in patients regardless of the study region, sample size, detection method, or analysis method, which further confirmed the prognostic potential of HMGA2. Subgroup analysis by cancer type revealed a significant correlation between HMGA2 expression and OS in gastric cancer, breast cancer, hepatocellular carcinoma, colorectal cancer, nasopharyngeal carcinoma, and esophageal carcinoma. However, no such correlation was seen in ovarian cancer. A study by Hetland et al. [53] has suggested that HMGA2 expression was unrelated to chemotherapy response or survival of ovarian serous carcinoma patients. Meanwhile, according to Califano et al. [22], overexpression of HMGA2 has no significant prognostic value for DFS and OS in multivariate analysis; even high HMGA2 expression combined with high body mass index (BMI; $\geq 25 \mathrm{~kg} / \mathrm{m}^{2}$ ) indicated a poor prognosis in patients with ovarian cancer. Our outcome is consistent

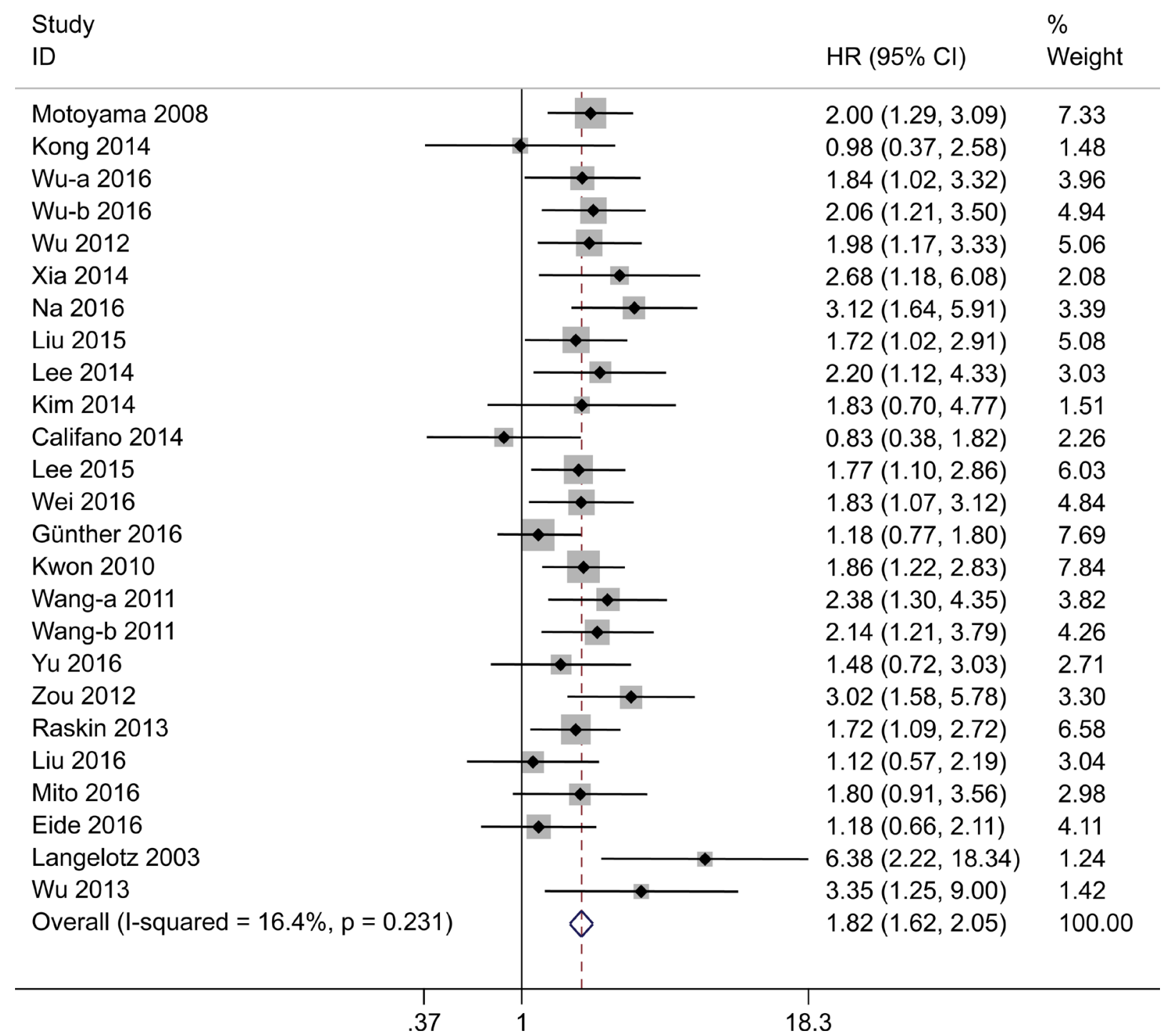

Figure 2: Forest plot to assess the association of HMGA2 expression with overall survival. 
Table 1: Correlation between HMGA2 expression and overall survival in cancer patients: subgroup analyses

\begin{tabular}{|c|c|c|c|c|c|c|c|c|}
\hline Categories & Studies & $\begin{array}{c}\text { Number of } \\
\text { patients }\end{array}$ & Model & HR (95\% CI) & $I^{2}(\%)$ & $P_{h}$ & $Z$ & $\boldsymbol{P}$ \\
\hline \multicolumn{9}{|l|}{ Cancer type } \\
\hline GC & 3 & 438 & Fixed & $1.77(1.31-2.41)$ & $0.0 \%$ & 0.418 & 3.67 & $<0.001$ \\
\hline $\mathrm{BC}$ & 3 & 652 & Fixed & $2.26(1.56-3.28)$ & $53.4 \%$ & 0.117 & 4.33 & $<0.001$ \\
\hline $\mathrm{HCC}$ & 2 & 441 & Fixed & $1.90(1.37-2.64)$ & $0.0 \%$ & 0.861 & 3.85 & $<0.001$ \\
\hline CRC & 4 & 547 & Fixed & $1.78(1.29-2.44)$ & $11.5 \%$ & 0.335 & 3.56 & $<0.001$ \\
\hline $\mathrm{OC}$ & 2 & 187 & Fixed & $1.14(0.62-2.09)$ & $36.3 \%$ & 0.210 & 0.42 & 0.673 \\
\hline NPC & 2 & 240 & Fixed & $1.96(1.26-3.05)$ & $0.0 \%$ & 0.372 & 3.00 & 0.003 \\
\hline $\mathrm{EC}$ & 2 & 187 & Fixed & $1.82(1.19-2.77)$ & $0.0 \%$ & 0.975 & 2.78 & 0.006 \\
\hline Other & 7 & 1062 & Random & $1.87(1.35-2.60)$ & $52.4 \%$ & 0.05 & 3.77 & $<0.001$ \\
\hline \multicolumn{9}{|l|}{ Study region } \\
\hline Asian & 17 & 2549 & Fixed & $1.95(1.69-2.25)$ & $0.0 \%$ & 0.764 & 9.16 & $<0.001$ \\
\hline Non-Asian & 8 & 1205 & Random & $1.60(1.14-2.25)$ & $51.3 \%$ & 0.045 & 3.18 & 0.001 \\
\hline \multicolumn{9}{|l|}{ Sample size } \\
\hline$\geq 100$ & 17 & 3156 & Fixed & $1.75(1.53-2.00)$ & $20.3 \%$ & 0.217 & 8.23 & $<0.001$ \\
\hline$<100$ & 8 & 598 & Fixed & $2.14(1.66-2.75)$ & $0.0 \%$ & 0.457 & 5.87 & $<0.001$ \\
\hline \multicolumn{9}{|l|}{$\begin{array}{l}\text { Detection } \\
\text { method }\end{array}$} \\
\hline $\mathrm{IHC}$ & 21 & 3085 & Fixed & $1.81(1.59-2.07)$ & $4.6 \%$ & 0.400 & 8.81 & $<0.001$ \\
\hline RT-PCR & 2 & 403 & Fixed & $1.69(1.19-2.18)$ & $0.0 \%$ & 0.591 & 6.66 & $<0.001$ \\
\hline qRT-PCR & 2 & 266 & Fixed & $1.66(1.17-2.34)$ & $50.7 \%$ & 0.154 & 2.84 & 0.005 \\
\hline \multicolumn{9}{|l|}{$\begin{array}{l}\text { Analysis } \\
\text { method }\end{array}$} \\
\hline Multivariate & 20 & 3145 & Fixed & $1.91(1.68-2.18)$ & $14.3 \%$ & 0.276 & 9.72 & $<0.001$ \\
\hline Univariate & 5 & 609 & Fixed & $1.49(1.14-1.96)$ & $0.0 \%$ & 0.408 & 2.89 & 0.004 \\
\hline
\end{tabular}

Notes: Random-effects model was used when $P$-value for heterogeneity test $<0.1$; otherwise, fixed-effects model was used. Abbreviations: GC: gastric cancer; BC: breast cancer; HCC: hepatocellular carcinoma; CRC: colorectal cancer; OC: ovarian cancer; NPC: nasopharyngeal carcinoma; EC: esophageal carcinoma; $P_{h}: P$-value for heterogeneity based on $Q$-test; $P: P$-value for statistical significance based on Z-test; HR: hazard ratio; IHC: immunohistochemistry; RT-PCR: reverse transcriptionpolymerase chain reaction; qRT-PCR: quantitative real-time polymerase chain reaction.

with results of these studies. However, there were only 2 studies included in this subgroup, so publication bias may have contributed to the negative outcome. Lymph node metastasis, lymphovascular space involvement, and advanced stage significantly affect survival outcomes of cancer patients [54]. According to our results, HMGA2 overexpression was significantly linked to advanced TNM stage (stage III/IV), positive lymphovascular space invasion, distant metastasis, and lymph node metastasis. This further verifies the prognostic role of HMGA2.

There are several limitations to this meta-analysis. First, only studies published in English were included. Since authors are more likely to publish positive results in an English-language journal [55], negative outcomes published in non-English journals may have been omitted from the analysis, leading to language bias. Second, differences in detection methods, interpretation of results, and parameter cut-off values among the included studies may have confounded the analyzed outcomes. In our included 29 studies, 13 studies defined the overexpression and low expression of HMGA2 by multiplying the scores of expression intensity and positivity area. Seven studies defined the overexpression and low expression by percentage of positive staining cells. Only one study defined the overexpression and low expression by the median HMGA2 mRNA expression level. Additionally, the rest eight studies did not report the cutoff values. All these studies reported that HMGA2 may be a promising prognostic factor for cancer patients. However, more studies are needed to reach a consensus for different methods and criteria for overexpression of HMGA2. Third, some studies did not provide OS or DFS/PFS/RFS data directly, which were subsequently extracted from KaplanMeier curves in such cases. This may have reduced 


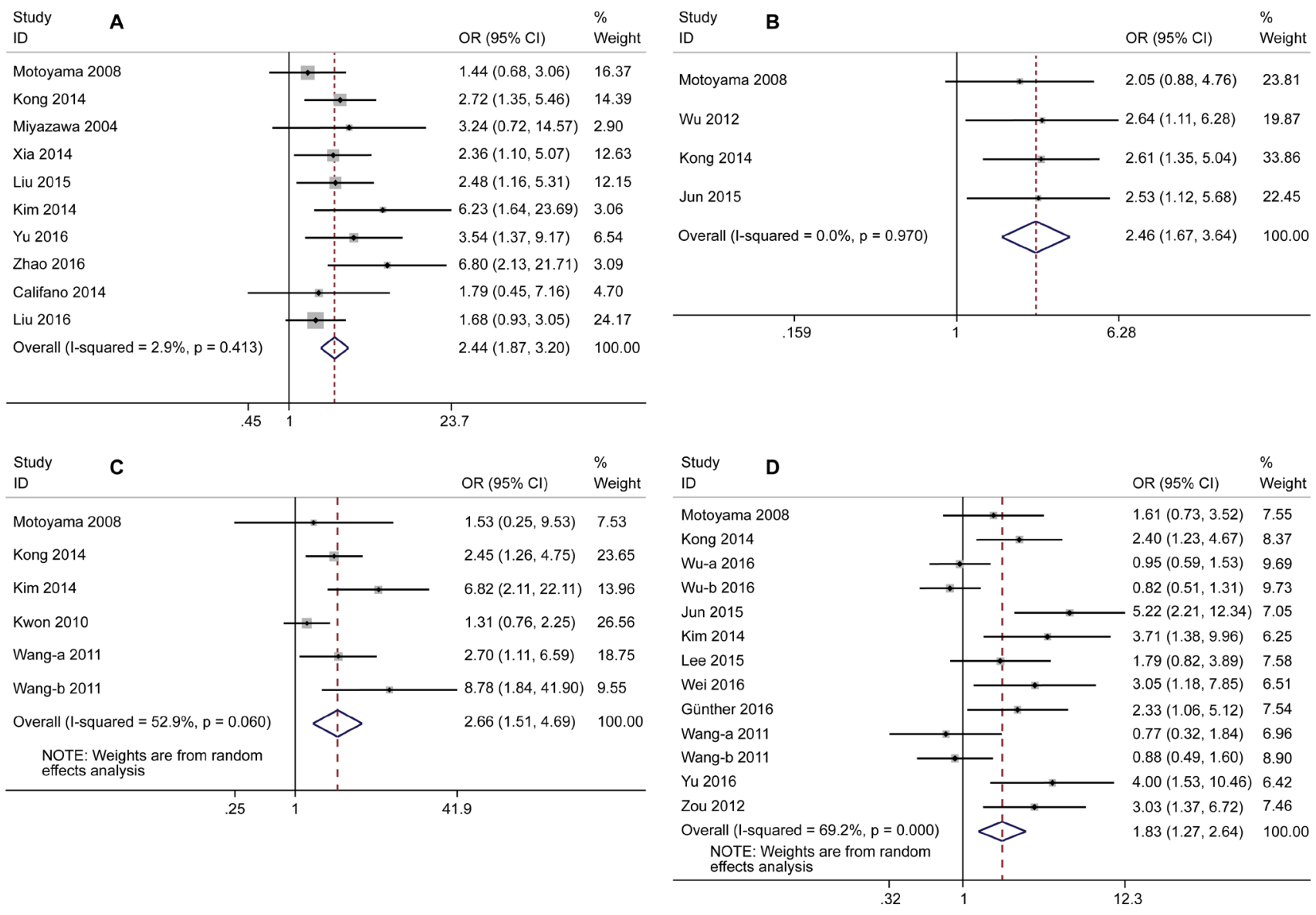

Figure 3: Forest plots of correlation between odds ratio and TNM stage (I/II vs. III/IV) (A), lymphovascular space invasion (negative vs. positive) (B), distant metastasis (absent $v s$. present) (C), lymph node metastasis (negative $v s$. positive) (D).

\begin{tabular}{|c|c|c|}
\hline \multirow{2}{*}{$\begin{array}{l}\text { Study } \\
\text { ID }\end{array}$} & \multirow[b]{2}{*}{$\mathrm{HR}(95 \% \mathrm{Cl})$} & \multirow{2}{*}{$\begin{array}{l}\% \\
\text { Weight }\end{array}$} \\
\hline & & \\
\hline Califano 2014 & $0.96(0.49,1.89)$ & 15.93 \\
\hline Jun 2015 & $3.20(1.50,6.79)$ & 14.54 \\
\hline Zhao 2016 & $\rightarrow 5.78(1.07,31.39)$ & 5.11 \\
\hline Yang 2011 & $3.47(1.43,8.43)$ & 12.34 \\
\hline Günther 2016 & $1.40(0.96,2.05)$ & 22.06 \\
\hline Liu 2016 & $1.22(0.69,2.16)$ & 18.06 \\
\hline Miyazawa 2004 & $3.48(1.40,8.69)$ & 11.95 \\
\hline Overall $(I-$ squared $=58.8 \%, p=0.024)$ & $1.94(1.27,2.98)$ & 100.00 \\
\hline NOTE: Weights are from random effects analysis & & \\
\hline
\end{tabular}

Figure 4: Forest plot to assess the association of HMGA2 expression with disease-free survival/progression-free survival/ recurrence-free survival. 
the credibility of the final results. Fourth, subgroup analysis could not eliminate heterogeneity across studies completely, which may have led to biases. Moreover, all the selected studies are small sample retrospective studies, which may cause reporting bias. Therefore, further larger, multi-center, high-quality prospective investigations are required to overcome the above-mentioned limitations.

\section{CONCLUSIONS}

In conclusion, this meta-analysis confirmed that high HMGA2 expression in cancer is linked to poor prognosis, and HMGA2 is a potential predictive biomarker for OS. Additional larger, well-designed multicenter prospective studies are necessary to confirm our results.

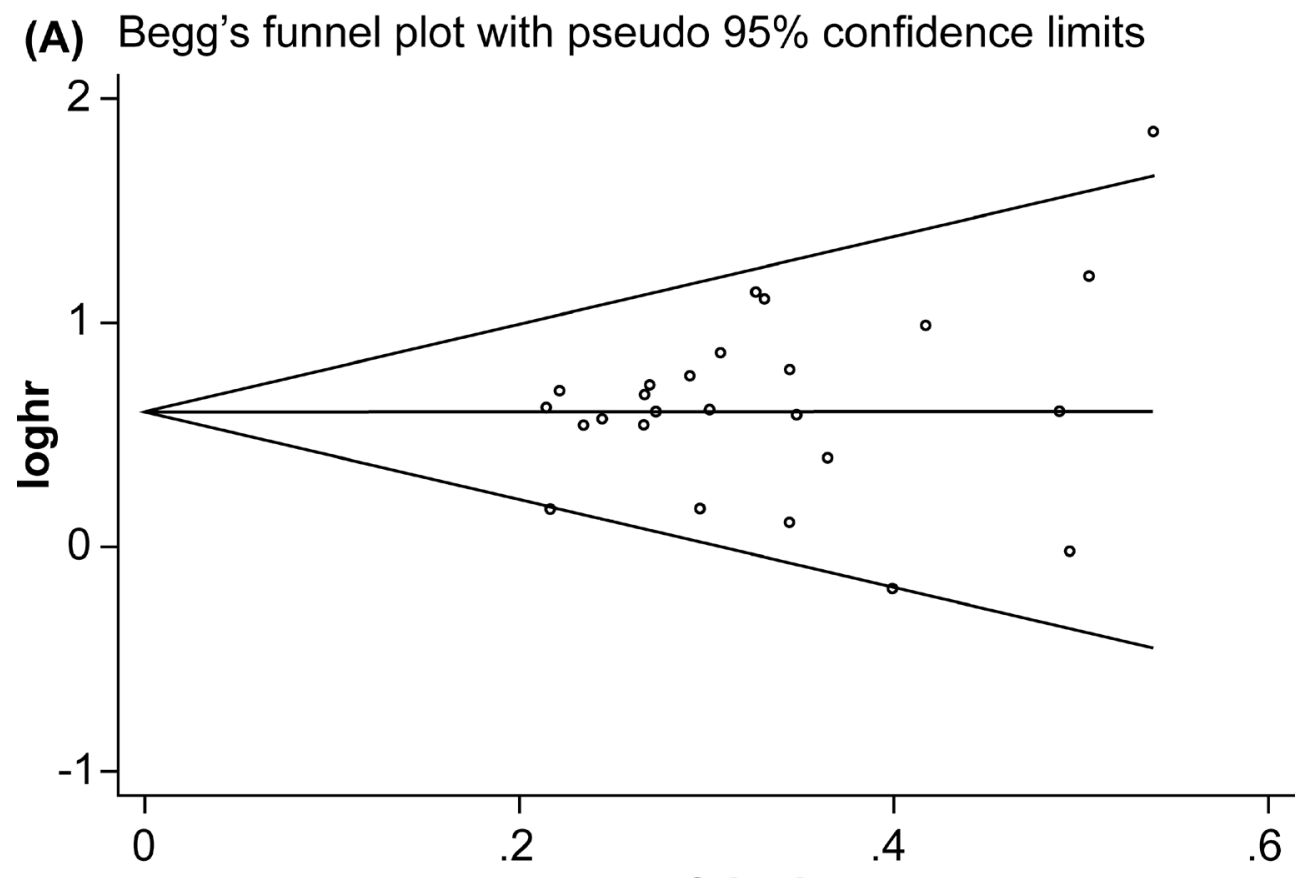

s.e. of: loghr

(B) Begg's funnel plot with pseudo 95\% confidence limits

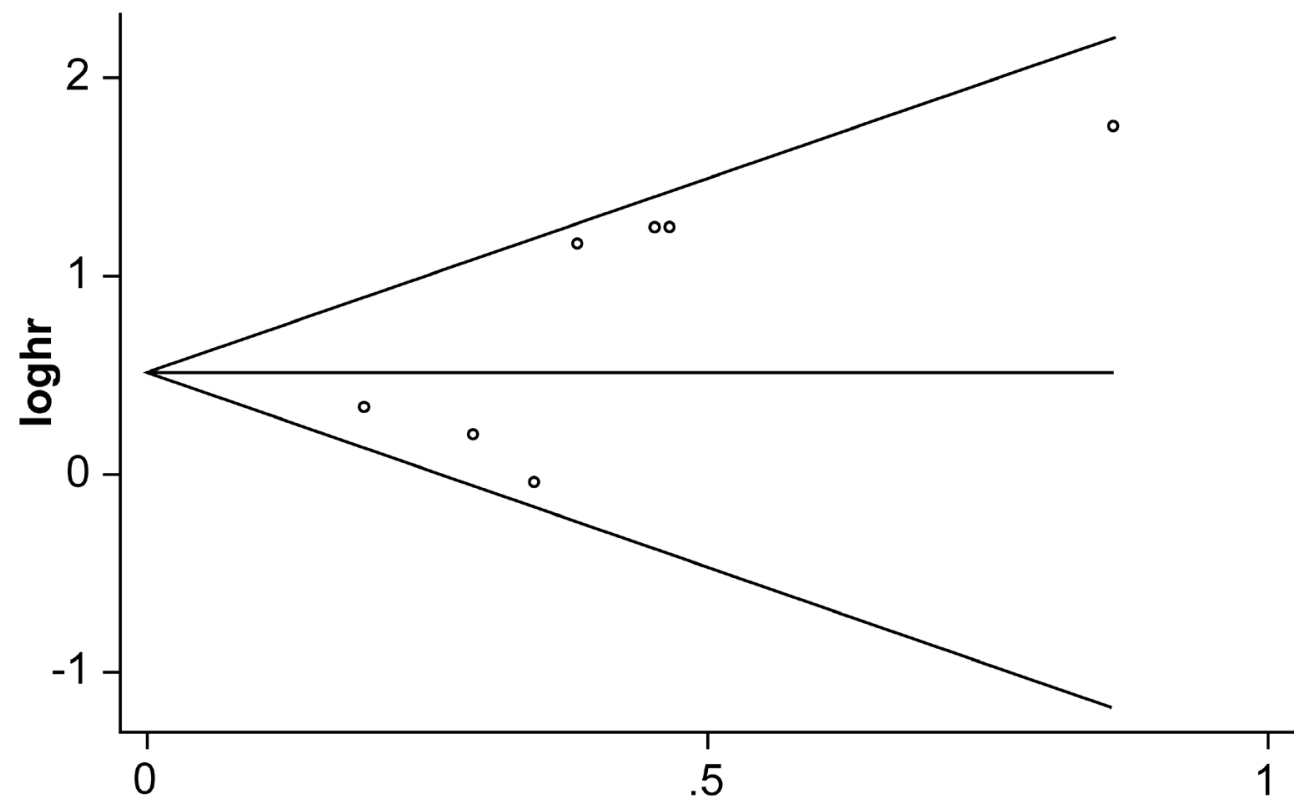

s.e. of: loghr

Figure 5: (A) Funnel plot for the meta-analysis of correlation between HMGA2 expression and overall survival. (B) Funnel plot for the meta-analysis of the correlation between HMGA2 expression and disease-free survival/progression-free survival/ recurrence-free survival. 


\section{MATERIALS AND METHODS}

\section{Search strategy}

A comprehensive search was performed in PubMed, EMBASE, and Web of Science databases to identify relevant articles published from 1996 to December 31, 2016. The search terms were as follows: "HMGA2 Protein", or "High-mobility group A2", or "HMGA2"), and ("neoplasm", or "cancer", or "carcinoma" or "tumor"), and ("prognosis", or "prognostic" or "outcome", or "survival"). We also retrieved relevant systematic reviews and references to find additional eligible studies. This meta-analysis complies with the guidelines of the Preferred Reporting Items for Systematic Reviews and Meta-Analyses (Supplementary Table 2) [56].

\section{Selection criteria}

Studies fitting the following inclusion criteria were analyzed: (1) correlation between the expression of HMGA2 and OS was assessed; (2) HMGA2 expression

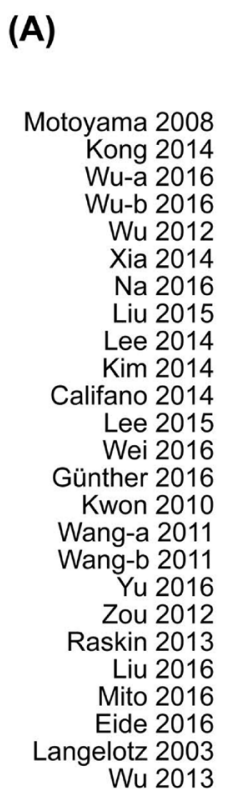

(B)

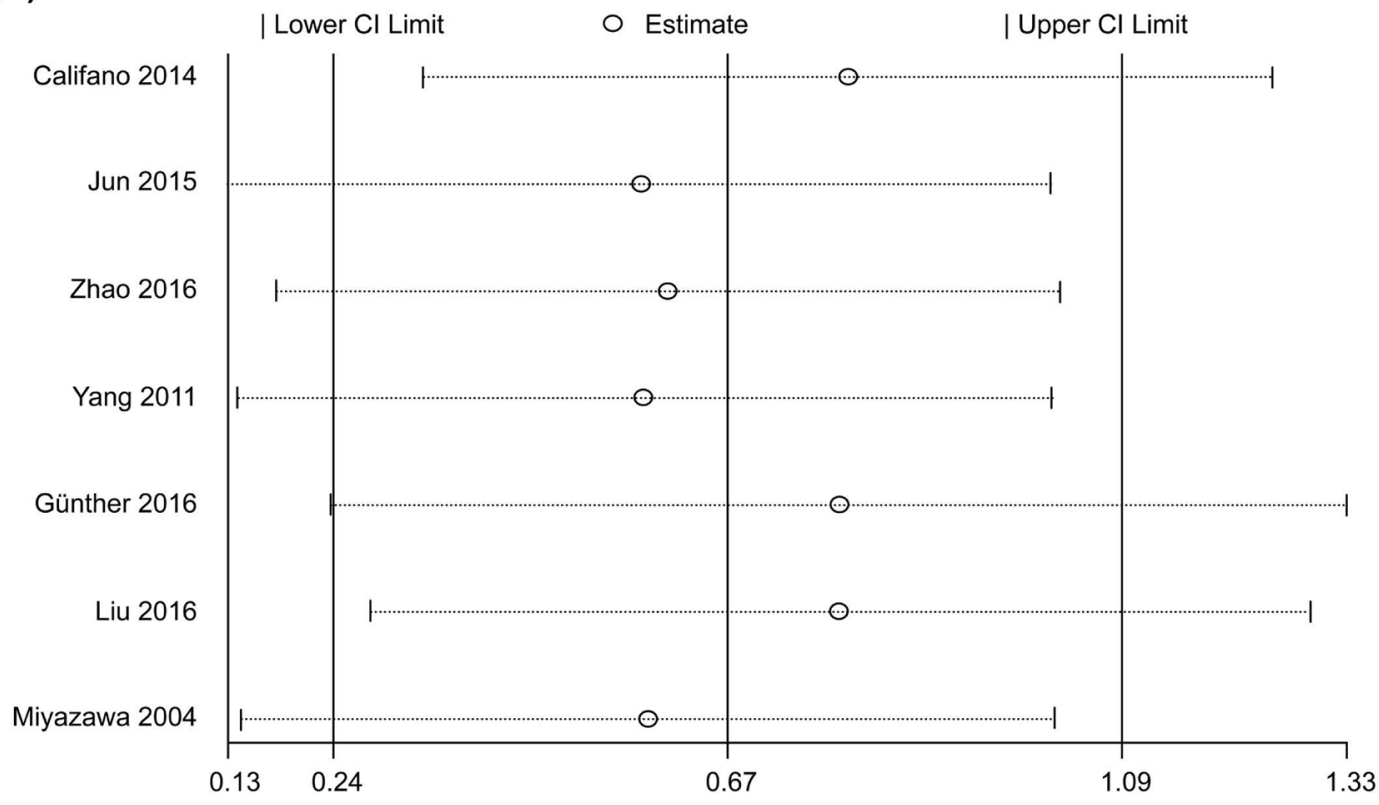

Figure 6: (A) Sensitivity analysis of the association between HMGA2 expression and overall survival. (B) Sensitivity analysis of the association between HMGA2 expression and disease-free survival/progression-free survival/recurrence-free survival. 
was analyzed by IHC, RT-PCR, or qRT-PCR; (3) patients were classified into high HMGA2 expression (or HMGA2positive) and low HMGA2 expression (or HMGA2negative) groups; (4) HR and 95\% CI were provided or could be extracted indirectly; (5) the article was written in English; (6) when publications involved the same patient population, only the largest patient cohort was included. Studies were excluded as per the following criteria: (1) were reviews, letters, involved basic research, or were animal studies; (2) provided inadequate survival data.

The articles included in the study were evaluated by two investigators (DN and LPZ), independently. Any disagreement between the investigators was resolved by consensus.

\section{Data extraction and quality assessment}

Two investigators (DN and LPZ) collected data from the selected articles independently. The author name(s), publication year, country, sample size, cancer type, clinic stage, HMGA2 detection method, follow-up time, and survival data including OS, DFS/PFS/RFS, were extracted. In studies including both univariate and multivariate analyses, only the HRs and the corresponding 95\% CIs from multivariate analyses were considered. Otherwise, the HRs and CIs were either extracted from univariate analysis, or were calculated from the KaplanMeier curve using methods provided in literature [57]. Study quality was evaluated according to the NOS and a score of six points or higher signified high quality.

\section{Statistical analysis}

The prognostic value of HMGA2 expression in predicting OS and PFS/RFS/DFS/ was evaluated by analyzing pooled HRs and respective $95 \%$ CIs. The pooled ORs and their 95\% CIs were combined to assess the correlation between HMGA2 expression and TNM stage (I/II vs. III/IV), lymphovascular space invasion (negative $v s$. positive), distant metastasis (absent vs. present), and lymph node metastasis (negative vs. positive). Chi-square test based on the $Q$ statistic and $I^{2}$ statistic was used for heterogeneity analysis. When significant heterogeneity was observed ( $P$-value $<0.1$ and/or $I^{2}>50 \%$ ), randomeffects model was used to analyze the pooled HRs. Otherwise, fixed-effects model was applied. Sensitivity analysis was conducted to validate the stability of the pooled outcomes. Publication bias was assessed using Begg's test and Egger's test. A $P$-value $<0.05$ was defined as statistically significance. All statistical analyses were performed using STATA 14.0 software (Stata Corporation, College Station, TX, USA).

\section{Abbreviations}

$\mathrm{BC}$, breast cancer; BMI, body mass index; CRC, colorectal cancer; DFS, disease-free survival; EC, esophageal carcinoma; EMT, epithelial-to-mesenchymal transition; GS, gastric cancer; HCC, hepatocellular; HMGA2, high mobility protein A2; HNSCC, head and neck squamous cell carcinoma; HR, hazard ratio; hTERT, human telomerase reverse transcriptase; IHC, immunohistochemistry; IncRNA, long non-coding ribonucleic acid; miRNAs, micro ribonucleic acids; NPC, nasopharyngeal carcinoma; OC, ovarian cancer; OR, odds ratio; OS, overall survival; PFS, progression-free survival; RFS, recurrence-free survival (RFS); RT-PCR, reverse transcription-polymerase chain reaction; TGF $\beta$, transforming growth factor beta.

\section{Author contributions}

Study conception and design: Dan Nie, Xiguang Mao; Data collection: Dan Nie, Lingping Zhang; Data analysis and interpretation: Dan Nie, Lingping Zhang, Qian Guo; Writing and revising of manuscript: Dan Nie, Xiguang Mao. All authors have reviewed the manuscript and approve of its submission.

\section{ACKNOWLEDGMENTS}

We would like to thank Editage (www.editage.com) for English language editing and Publication Support.

\section{CONFLICTS OF INTEREST}

The authors declare no conflicts of interest.

\section{FUNDING}

This study was supported by grants from the Health and Family Planning Commission of Sichuan Province (No. 130374), the Science and Technology Bureau of Luzhou (No. 2013-S-48(15/30)), and the Southwest Medical University Youth Research Fund (No. 2014QN-06).

\section{REFERENCES}

1. Chen W, Zheng R, Baade PD, Zhang S, Zeng H, Bray F, Jemal A, Yu XQ, He J. Cancer statistics in China, 2015. CA Cancer J Clin. 2016; 66:115-132.

2. Torre LA, Bray F, Siegel RL, Ferlay J, Lortet-Tieulent J, Jemal A. Global cancer statistics, 2012. CA Cancer J Clin. 2015; 65:87-108.

3. Miller KD, Siegel RL, Lin CC, Mariotto AB, Kramer JL, Rowland JH, Stein KD, Alteri R, Jemal A. Cancer treatment and survivorship statistics, 2016. CA Cancer J Clin. 2016; 66:271-289.

4. Fan YH, Yan F, Chai Y, Jiang Y, Zhu X, Wang R. Long noncoding RNA HOTTIP as an independent prognostic marker in cancer. Clin Chim Acta. 2017 Aug 2. https://doi. org/10.1016/j.cca.2017.07.031. 
5. Young AR, Narita M. Oncogenic HMGA2: short or small. Genes Dev. 2007; 21:1005-1009.

6. Fusco A, Fedele M. Roles of HMGA proteins in cancer. Nat Rev Cancer. 2007; 7:899-910.

7. Cleynen I, Van de Ven WJ. The HMGA proteins: a myriad of functions (Review). Int J Oncol. 2008; 32:289-305.

8. Fedele M, Fusco A. HMGA and cancer. Biochim Biophys Acta. 2010; 1799:48-54.

9. Wu J, Wei JJ. HMGA2 and high-grade serous ovarian carcinoma. J Mol Med (Berl). 2013; 91:1155-1165.

10. Wu J, Zhang S, Shan J, Hu Z, Liu X, Chen L, Ren X, Yao L, Sheng H, Li L, Ann D, Yen Y, Wang J, et al. Elevated HMGA2 expression is associated with cancer aggressiveness and predicts poor outcome in breast cancer. Cancer Lett. 2016; 376:284-292.

11. Wu L, Wang Z, Lu R, Jiang W. Expression of high mobility group A2 is associated with poor survival in hepatocellular carcinoma. Pathol Oncol Res. 2012; 18:983-987.

12. Na N, Si T, Huang Z, Miao B, Hong L, Li H, Qiu J, Qiu J. High expression of HMGA2 predicts poor survival in patients with clear cell renal cell carcinoma. Onco Targets Ther. 2016; 9:7199-7205.

13. Kwon JH, Kim J, Park JY, Hong SM, Park CW, Hong SJ, Park SY, Choi YJ, Do IG, Joh JW, Kim DS, Choi KY. Overexpression of high-mobility group box 2 is associated with tumor aggressiveness and prognosis of hepatocellular carcinoma. Clin Cancer Res. 2010; 16:5511-5521.

14. Wang X, Liu X, Li AY, Chen L, Lai L, Lin HH, Hu S, Yao L, Peng J, Loera S, Xue L, Zhou B, Zhou L, et al. Overexpression of HMGA2 promotes metastasis and impacts survival of colorectal cancers. Clin Cancer Res. 2011; 17:2570-2580.

15. Zhu J, Wang $\mathrm{H}, \mathrm{Xu} \mathrm{S}$, Hao Y. Clinicopathological and prognostic significance of HMGA2 overexpression in gastric cancer: a meta-analysis. Oncotarget. 2017; 8:100478100489. https://doi.org/10.18632/oncotarget.19001.

16. Motoyama K, Inoue H, Nakamura Y, Uetake H, Sugihara K, Mori M. Clinical significance of high mobility group A2 in human gastric cancer and its relationship to let-7 microRNA family. Clin Cancer Res. 2008; 14:2334-2340.

17. Lee J, Ha S, Jung CK, Lee HH. High-mobility-group A2 overexpression provokes a poor prognosis of gastric cancer through the epithelial-mesenchymal transition. Int J Oncol. 2015; 46:2431-2438.

18. Jun KH, Jung JH, Choi HJ, Shin EY, Chin HM. HMGA1/ HMGA2 protein expression and prognostic implications in gastric cancer. Int J Surg. 2015; 24:39-44.

19. Langelotz C, Schmid P, Jakob C, Heider U, Wernecke KD, Possinger K, Sezer O. Expression of high-mobilitygroup-protein HMGI-C mRNA in the peripheral blood is an independent poor prognostic indicator for survival in metastatic breast cancer. Br J Cancer. 2003; 88:1406-1410.

20. Yu FY, Tu Y, Deng Y, Guo C, Ning J, Zhu Y, Lv X, Ye H. MiR-4500 is epigenetically downregulated in colorectal cancer and functions as a novel tumor suppressor by regulating HMGA2. Cancer Biol Ther. 2016; 17:1149-1157.

21. Liu TP, Huang CC, Yeh KT, Ke TW, Wei PL, Yang JR, Cheng YW. Down-regulation of let-7a-5p predicts lymph node metastasis and prognosis in colorectal cancer: Implications for chemotherapy. Surg Oncol. 2016; 25:429-434.

22. Califano D, Pignata S, Losito NS, Ottaiano A, Greggi S, De Simone V, Cecere S, Aiello C, Esposito F, Fusco A, Chiappetta G. High HMGA2 expression and high body mass index negatively affect the prognosis of patients with ovarian cancer. J Cell Physiol. 2014; 229:53-59.

23. Kim TH, Song JY, Park H, Jeong JY, Kwon AY, Heo JH, Kang H, Kim G, An HJ. miR-145, targeting high-mobility group A2, is a powerful predictor of patient outcome in ovarian carcinoma. Cancer Lett. 2015; 356:937-945.

24. Liu Z, Wu K, Yang Z, Wu A. High-mobility group A2 overexpression is an unfavorable prognostic biomarker for nasopharyngeal carcinoma patients. Mol Cell Biochem. 2015; 409:155-162.

25. Xia YY, Yin L, Tian H, Guo WJ, Jiang N, Jiang XS, Wu $\mathrm{J}$, Chen M, Wu JZ, He X. HMGA2 is associated with epithelial-mesenchymal transition and can predict poor prognosis in nasopharyngeal carcinoma. Onco Targets Ther. 2015; 8:169-176.

26. Mito J, Agoston A, Dal Cin P, Srivastava A. HMGA2 Expression Correlates with High Tumor Stage, Nodal Metastasis, and Poor Outcomes in Patients with Esophageal Adenocarcinoma. Laboratory Investigation. Nature Publishing Group. 2016; 96:187A.

27. Mito J, Agoston A, Dal Cin P, Srivastava A. (2016, February). HMGA2 Expression Correlates with High Tumor Stage, Nodal Metastasis, and Poor Outcomes in Patients with Esophageal Adenocarcinoma. In Laboratory Investigation (Vol. 96, pp. 187A-187A). 75 Varick St, 9th Fl, New York, NY 10013-1917 USA: Nature Publishing Group.

28. Zhao XP, Zhang H, Jiao JY, Tang DX, Wu YL, Pan CB. Overexpression of HMGA2 promotes tongue cancer metastasis through EMT pathway. J Transl Med. 2016; $14: 26$.

29. Miyazawa J, Mitoro A, Kawashiri S, Chada KK, Imai K. Expression of mesenchyme-specific gene HMGA2 in squamous cell carcinomas of the oral cavity. Cancer Res. 2004; 64:2024-2029.

30. Gunther K, Foraita R, Friemel J, Gunther F, Bullerdiek J, Nimzyk R, Markowski DN, Behrens T, Ahrens W. The Stem Cell Factor HMGA2 Is Expressed in Non-HPV-Associated Head and Neck Squamous Cell Carcinoma and Predicts Patient Survival of Distinct Subsites. Cancer Epidemiol Biomarkers Prev. 2017; 26:197-205.

31. Lee CT, Wu TT, Lohse CM, Zhang L. High-mobility group AT-hook 2: an independent marker of poor prognosis in intrahepatic cholangiocarcinoma. Hum Pathol. 2014; 45:2334-2340.

32. Wu ZB, Cai L, Lin SJ, Xiong ZK, Lu JL, Mao Y, Yao Y, Zhou LF. High-mobility group box 2 is associated with 
prognosis of glioblastoma by promoting cell viability, invasion, and chemotherapeutic resistance. Neuro Oncol. 2013; 15:1264-1275.

33. Zou Q, Xiong L, Yang Z, Lv F, Yang L, Miao X. Expression levels of HMGA2 and CD9 and its clinicopathological significances in the benign and malignant lesions of the gallbladder. World J Surg Oncol. 2012; 10:92.

34. Raskin L, Fullen DR, Giordano TJ, Thomas DG, Frohm ML, Cha KB, Ahn J, Mukherjee B, Johnson TM, Gruber SB. Transcriptome profiling identifies HMGA2 as a biomarker of melanoma progression and prognosis. J Invest Dermatol. 2013; 133:2585-2592.

35. Eide HA, Halvorsen AR, Bjaanæs MM, Piri H, Holm R, Solberg S, Jørgensen L, Brustugun OT, Kiserud CE, Helland $\AA$. The MYCN-HMGA2-CDKN2A pathway in non-small cell lung carcinoma--differences in histological subtypes. BMC Cancer. 2016; 16:71.

36. Yang GL, Zhang LH, Bo JJ, Hou KL, Cai X, Chen YY, Li H, Liu DM, Huang YR. Overexpression of HMGA2 in bladder cancer and its association with clinicopathologic features and prognosis HMGA2 as a prognostic marker of bladder cancer. Eur J Surg Oncol. 2011; 37:265-271.

37. Pallante P, Sepe R, Puca F, Fusco A. High mobility group a proteins as tumor markers. Front Med (Lausanne). 2015; 2:15.

38. Li AY, Lin HH, Kuo CY, Shih HM, Wang CC, Yen Y, Ann DK. High-mobility group A2 protein modulates hTERT transcription to promote tumorigenesis. Mol Cell Biol. 2011; 31:2605-2617.

39. Wu J, Wang Y, Xu X, Cao H, Sahengbieke S, Sheng H, Huang Q, Lai M. Transcriptional activation of FN1 and IL11 by HMGA2 promotes the malignant behavior of colorectal cancer. Carcinogenesis. 2016; 37:511-521.

40. Morishita A, Zaidi MR, Mitoro A, Sankarasharma D, Szabolcs M, Okada Y, D'Armiento J, Chada K. HMGA2 is a driver of tumor metastasis. Cancer Res. 2013; 73:4289-4299.

41. Zha L, Zhang J, Tang W, Zhang N, He M, Guo Y, Wang Z. HMGA2 elicits EMT by activating the Wnt/ $\beta$-catenin pathway in gastric cancer. Dig Dis Sci. 2013; 58:724-733.

42. Dong J, Wang R, Ren G, Li X, Wang J, Sun Y, Liang J, Nie Y, Wu K, Feng B, Shang Y, Fan D. HMGA2-FOXL2 Axis Regulates Metastases and Epithelial-to-mesenchymal Transition of Chemo-resistant Gastric Cancer. Clin Cancer Res. 2017; 23:3461-73. https://doi.org/10.1158/1078-0432. CCR-16-2180.

43. Sun $M$, Song $C X$, Huang $H$, Frankenberger $C A$, Sankarasharma D, Gomes S, Chen P, Chen J, Chada KK, He C, Rosner MR. HMGA2/TET1/HOXA9 signaling pathway regulates breast cancer growth and metastasis. Proc Natl Acad Sci USA. 2013; 110:9920-9925.

44. Liu W, Xu G, Liu H, Li T. MicroRNA-490-3p regulates cell proliferation and apoptosis by targeting HMGA2 in osteosarcoma. FEBS Lett. 2015; 589:3148-3153.

45. Li T, Yang XD, Ye CX, Shen ZL, Yang Y, Wang B, Guo P, Gao ZD, Ye YJ, Jiang KW, Wang S. Long noncoding
RNA HIT000218960 promotes papillary thyroid cancer oncogenesis and tumor progression by upregulating the expression of high mobility group AT-hook 2 (HMGA2) gene. Cell Cycle. 2017; 16:224-231.

46. Strell C, Norberg KJ, Mezheyeuski A, Schnittert J, Kuninty PR, Moro CF, Paulsson J, Schultz NA, Calatayud D, Löhr JM, Frings O, Verbeke CS, Heuchel RL, et al. Stromaregulated HMGA2 is an independent prognostic marker in PDAC and AAC. Br J Cancer. 2017; 117:65-77.

47. Ren W, Gao L, Li F, Qiang C, Li S, Zheng J, Kong X, Deng J, Cai G, Zhang H, Zhou M, Zhi K. Circulating HMGA2 and PLAG1 in blood of patients with oral squamous cell carcinoma. J Oral Pathol Med. 2017; 46:998-1003. https:// doi.org/10.1111/jop.12609.

48. Bush BM, Brock AT, Deng JA, Nelson RA, Sumter TF. The Wnt/ $\beta$-catenin/T-cell factor 4 pathway up-regulates high-mobility group A1 expression in colon cancer. Cell Biochem Funct. 2013; 31:228-236.

49. Shah SN, Cope L, Poh W, Belton A, Roy S, Talbot CC, Sukumar S, Huso DL, Resar LM. HMGA1: a master regulator of tumor progression in triple-negative breast cancer cells. PLoS One. 2013; 8:e63419.

50. Zhang Z, Wang Q, Chen F, Liu J. Elevated expression of HMGA1 correlates with the malignant status and prognosis of non-small cell lung cancer. Tumour Biol. 2015; 36:1213-1219.

51. Hristov AC, Cope L, Di CF, Reyes MD, Singh M, Hillion JA, Belton A, Joseph B, Schuldenfrei A, IacobuzioDonahue CA, Maitra A, Resar LM. HMGA1 correlates with advanced tumor grade and decreased survival in pancreatic ductal adenocarcinoma. Mod Pathol. 2010; 23:98-104.

52. Liau SS, Rocha F, Matros E, Redston M, Whang E. High mobility group AT-hook 1 (HMGA1) is an independent prognostic factor and novel therapeutic target in pancreatic adenocarcinoma. Cancer. 2008; 113:302-314.

53. Hetland TE, Holth A, Kærn J, Flørenes VA, Tropé CG, Davidson B. HMGA2 protein expression in ovarian serous carcinoma effusions, primary tumors, and solid metastases. Virchows Arch. 2012; 460:505-513.

54. Peiretti M, Zapardiel I, Zanagnolo V, Landoni F, Morrow CP, Maggioni A. Management of recurrent cervical cancer: a review of the literature. Surg Oncol. 2012; 21:e59-e66.

55. Egger $\mathrm{M}$, Zellweger-Zähner $\mathrm{T}$, Schneider $\mathrm{M}$, Junker C, Lengeler C, Antes G. Language bias in randomised controlled trials published in English and German. Lancet. 1997; 350:326-329.

56. Moher D, Liberati A, Tetzlaff J, Altman DG, and PRISMA Group. Preferred reporting items for systematic reviews and meta-analyses: the PRISMA statement. BMJ. 2009; 339:b2535.

57. Tierney JF, Stewart LA, Ghersi D, Burdett S, Sydes MR. Practical methods for incorporating summary time-to-event data into meta-analysis. Trials. 2007; 8:16. 